\title{
THE $q$-ANALOGUE OF THE $p$-ADIC GAMMA FUNCTION
}

\author{
By HaJime NaKaZATo
}

\section{Introduction.}

The $p$-adic gamma function $\Gamma_{p}(x)$ was defined and studied by Morita [9] and the $p$-adic log-gamma function $G_{p}(x)$ was defined and studied by Diamond [3]. The Morita's gamma function $\Gamma_{p}(x)$ is defined by

$$
\Gamma_{p}(x)=\lim _{\substack{n \rightarrow x \\ \ln \boldsymbol{Z}_{p}}}(-1)^{n} \prod_{0<j<n}^{*} j \quad \text { for } \quad x \in \boldsymbol{Z}_{p},
$$

where $n$ runs over positive integers and $\Pi^{*}$ means that indices $j$ divisivle by $p$ are omitted. The Diamond's log-gamma function $G_{p}(x)$ and $G_{p}^{*}(x)$ are defined by

and

$$
G_{p}(x)=\lim _{n \rightarrow \infty} \frac{1}{p^{n}} \sum_{0 \leq j<p}(x+j)\{\log (x+j)-1\} \quad \text { for } \quad x \in \boldsymbol{C}_{p}-\boldsymbol{Z}_{p}
$$

$$
G_{p}^{*}(x)=\lim _{n \rightarrow \infty} \frac{1}{p^{n}} \sum_{0 \leq j<p}^{*}(x+j)\{\log (x+j)-1\} \quad \text { for } \quad x \in C_{p}-Z_{p}^{*},
$$

where $\log$ is the Iwasawa $p$-adic logarithm [5], $\boldsymbol{C}_{p}$ denotes the completion of the algebraic closure of the $p$-adic number field $\boldsymbol{Q}_{p}$ and $\Sigma^{*}$ means that indices $j$ divisible by $p$ are omitted in the summation.

Then $G_{p}(x)$ and $G_{p}^{*}(x)$ have the following two connections with $\Gamma_{p}(x)$.

THEOREM (Diamond [3], Ferrero-Greenberg [4]).

$$
\begin{aligned}
& \log \Gamma_{p}(x)=G_{p}^{*}(x) \quad \text { for } \quad x \in p \boldsymbol{Z}_{p} . \\
& \log \Gamma_{p}(x)=\sum_{\substack{0 \leq i<p \\
x+i \in \boldsymbol{Z}_{p}^{*}}} G_{p}\left(\frac{x+i}{p}\right) \quad \text { for } \quad x \in \boldsymbol{Z}_{p} .
\end{aligned}
$$

A generalized $p$-adic gamma function $\Gamma_{p, q}(x)$, depending on a parameter $q \in C_{p}$ with $|q-1|_{p}<1$ and $q \neq 1$, was defined and studied by Koblitz [7], [8]. We recall that the Koblitz' function $\Gamma_{p, q}(x)$ is defined by

$$
\Gamma_{p, q}(x)=\lim _{\substack{n \rightarrow x \\ \ln \boldsymbol{Z}_{p}}}(-1)^{n} \prod_{\substack{0<j<n \\ 0}} \frac{1-q^{j}}{1-q} \quad \text { for } \quad x \in \boldsymbol{Z}_{p},
$$

Received December 17, 1987 
where $n$ runs over positive integers.

As for the log-gamma functions $G_{p}(x)$ and $G_{p}^{*}(x)$ Koblitz defined only the $p$-adic psi-function $\phi_{p, q}(x)$ and $\phi_{p, q}^{*}(x)$, which are analogues of the derivatives

$$
\psi_{p}(x)=\frac{d}{d x} G_{p}(x) \text { and } \psi_{p}^{*}(x)=\frac{d}{d x} G_{p}^{*}(x) .
$$

For $q \in C_{p}$ such that $|q-1|_{p}<1$ and $\log (q) \neq 0$, let

$$
r(q)=\frac{|p|_{p}^{1 /(p-1)}}{|\log (q)|_{p}}
$$

Let $\quad d(x)=\min _{u \in Z_{p}}|x-u|_{p} \quad$ and $\quad d^{*}(x)=\min _{u \in Z_{p}^{*}}|x-u|_{p} \quad$ for $\quad x \in \boldsymbol{C}_{p}$.

Let $D(q)=\left\{x \in \boldsymbol{C}_{p} \mid 0<d(x)<r(q)\right\} \quad$ and

$D^{*}(q)=\left\{x \in \boldsymbol{C}_{p} \mid 0<d^{*}(x)<r(q)\right\}$.

Putting

and

$$
\psi_{p, q}(x)=\lim _{n \rightarrow \infty} \frac{1}{p^{n}} \sum_{0 \leqq j<p n} \log \frac{1-q^{x+\jmath}}{1-q} \quad \text { for } \quad x \in D(q)
$$

$$
\phi_{p, q}^{*}(x)=\lim _{n \rightarrow \infty} \frac{1}{p^{n}} \sum_{0 \leq j<p^{n}} \log \frac{1-q^{x+\jmath}}{1-q} \quad \text { for } \quad x \in D^{*}(q),
$$

Koblitz [7] gave the following

THEOREM.

$$
\begin{aligned}
& \frac{d}{d x} \log \Gamma_{p, q}(x)=\psi_{p, q}^{*}(x) \quad \text { for } \quad x \in p \boldsymbol{Z}_{p} . \\
& \frac{d}{d x} \log \Gamma_{p, q}(x)=\frac{1}{p} \sum_{\substack{0 \leq i<p \\
x+i \in Z_{p}^{*}}} \phi_{p, q}\left(\frac{x+i}{p}\right)+\left(1-\frac{1}{p}\right) \log \frac{1-q^{p}}{1-q} \quad \text { for } \quad x \in \boldsymbol{Z}_{p} .
\end{aligned}
$$

The purpose of this paper is to construct and study natural analogues $G_{p, q}(x)$ and $G_{p, q}^{*}(x)$ of the $p$-adic log-gamma functions $G_{p}(x)$ and $G_{p}^{*}(x)$, which have connections with $\Gamma_{p, q}(x)$.

Let $l_{2}(z)$ be the $p$-adic dilogarithm defined and studied by Coleman [2]. For a positive integer $n$, let $\tilde{n}=[(n-1) / p]+1$ where [ ] means Gauss symbol. Then the map extends to a continuous function on $\boldsymbol{Z}_{p}$ with values in $\boldsymbol{Z}_{p}$ (See [7]). Since $l_{2}(z)$ is locally analytic on $C_{p}-\{1\}$. Using Diamond's theorem [3], we may define analogues $G_{p, q}(x)$ and $G_{p, q}^{*}(x)$ of the log-gamma functions $G_{p}(x)$ and $G_{p}^{*}(x)$ by

$$
G_{p, q}(x)=\lim _{n \rightarrow \infty} \frac{1}{p^{n}} \sum_{0 \leq j<p}\left\{-\frac{1}{\log (q)} l_{2}\left(q^{x+j}\right)-(x+j) \log (1-q)\right\}
$$

for $x \in D(q)$ and 


$$
G_{p, q}^{*}(x)=\lim _{n \rightarrow \infty} \frac{1}{p^{n}} \sum_{0 \leqq j<p^{n}}^{*}\left\{-\frac{1}{\log (q)} l_{2}\left(q^{x+j}\right)-(x+j) \log (1-q)\right\}
$$

for $x \in D^{*}(q)$. Then we obtain

THEOREM (3.1). Suppose $|q-1|_{p}<|p|_{p}^{1 /(p-1)}$.

$$
\begin{aligned}
& \log \Gamma_{p, q}(x)=G_{p, q}^{*}(x)+\frac{p-1}{24} \log (q) \quad \text { for } \quad x \in p \boldsymbol{Z}_{p} . \\
& \log \Gamma_{p, q}(x)=\sum_{\substack{0 \leq i<p \\
x+i \in \mathbb{Z}_{p}^{*}}}\left\{G_{p, q p}\left(\frac{x+i}{p}\right)+\frac{\log (q)}{24}\right\}+(x-\tilde{x}) \log \frac{1-q^{p}}{1-q}
\end{aligned}
$$

for $x \in Z_{p}$.

Remark. By the definition of $\sim$ we have

$$
\frac{d}{d x}(\tilde{x})=\lim _{n \rightarrow \infty} \frac{\left(x+p^{n}\right)^{\sim}-x^{\sim}}{p^{n}}=\frac{1}{p} \quad \text { for } \quad x \in Z_{p},
$$

Differentiating in the equations of our theorem in the above sense we have the equations of the Koblitz' theorem.

For $G_{p, q}(x)$ and $G_{p, q}^{*}(x)$ we have the difference equations (2.3), the multiplication-theorem (2.6) and the reflection formula (2.4).

Remark. It is possible to define and study "twisted" functions of our $p$ adic gamma functions.

\section{Notation and definition.}

Let $\boldsymbol{Q}$ be the rational number field and let $\boldsymbol{Z}$ be the integer ring. Let $p$ be an odd prime. Let $\boldsymbol{Q}_{p}, \boldsymbol{Z}_{p}$ and $\boldsymbol{C}_{p}$ be the $p$-adic number field, the $p$-adic integer ring and the $p$-adic completion of the algebraic closure of $\boldsymbol{Q}_{p}$. Let $|x|_{p}$ be the absolute value of $x \in C_{p}^{*}$ such that $|p|_{p}=p^{-1}$.

Let $\log (u)=\log _{p}(u)$ be the Iwasawa $p$-adic logarithm [5] on $u \in C_{p}^{*}$. Then we have

$$
\log (u)=\sum_{n \geq 1}(-1)^{n-1} \frac{1}{n}(u-1)^{n} \quad \text { for } \quad|u-1|_{p}<1 .
$$

Let $\exp (u)=\exp _{p}(u)$ be the $p$-adic exponential function defined by

$$
\exp (u)=\sum_{n \geqq 0} \frac{1}{n !} u^{n} \quad \text { for } \quad|u|_{p}<|p|_{p}^{1 /(p-1)} .
$$

Let $l_{2}(u)$ be the $p$-adic dilogarithm [2] on $u \in C_{p}$ with $u \neq 1$. Then we have

$$
l_{2}(u)=\sum_{n \geqq 1} \frac{1}{n^{2}} u^{n} \quad \text { for } \quad|u|_{p}<1 .
$$


We assume hereafter that $q \in \boldsymbol{C}_{p}$ with $|q-1|_{p}<|p|_{p}^{1 /(p-1)}$ and $q \neq 1$. Then we have [7]

and

$$
\begin{aligned}
& r(q)=\frac{|p|_{p}^{1 /(p-1)}}{|\log (q)|_{p}}=\frac{|p|_{p}^{1 /(p-1)}}{|1-q|_{p}}>1, \\
& D(q)=\left\{x \in C_{p}-\left.Z_{p}|| x\right|_{p}<r(q)\right\}
\end{aligned}
$$

$$
D^{*}(q)=\left\{x \in \boldsymbol{C}_{p}-\left.\boldsymbol{Z}_{p}^{*}|| x\right|_{p}<r(q)\right\} .
$$

Let $q^{u}=\exp (u \cdot \log (q))$ for $|u|_{p}<r(q)$. Then

$$
\log \left(q^{u}\right)=u \cdot \log (q) \quad \text { for } \quad|u|_{p}<r(q) .
$$

1. Definition of $G_{p, q}(x)$ and $G_{p, q}^{*}(x)$.

Let

$$
L_{2, q}(u)=-\frac{1}{\log (q)} l_{2}\left(q^{u}\right)-u \cdot \log (1-q) \quad \text { for } \quad|u|_{p}<r(q), u \neq 0 .
$$

where $l_{2}(x)$ is the $p$-adic dilogarithm [2] and log is the $p$-adic logarithm normalized by $\log (p)=0[5]$.

Using the functional equation

$$
l_{2}(x)+l_{2}(1-x)=\log (x) \log (1-x) \quad \text { for } \quad x \neq 0 \text { and } x \neq 1,
$$

we have

$$
L_{2, q}(u)=\frac{1}{\log (q)} l_{2}\left(1-q^{u}\right)+u \cdot \log \frac{1-q^{u}}{1-q} \quad \text { for } \quad|u|_{p}<r(q), u \neq 0 .
$$

Since

$$
\begin{aligned}
l_{2}\left(1-q^{u}\right) & =1-q^{u}+(1 / 4)\left(1-q^{u}\right)^{2}+\cdots \\
& =-u \cdot \log (q)+(\log (q))^{2}\left\{-u^{2} / 2+u^{2} / 4+\cdots\right\} .
\end{aligned}
$$

We have

$$
\lim _{q \rightarrow 1} L_{2, q}(u)=-u+u \cdot \log (u) .
$$

Lemma (1.1). (1) $L_{2, q}(u)$ is locally analytic on: $u \in C_{p}$ with $|u|_{p}<r(q)$ and $u \neq 0$.

$$
\begin{aligned}
& \frac{d}{d u} L_{2, q}(u)=\log \frac{1-q^{u}}{1-q} . \\
& L_{2, q}(u)+L_{2, q}(-u)=\frac{1}{2} u^{2} \cdot \log (q) . \\
& L_{2, q}(u)+L_{2, q-1}(-u)=-u \cdot \log (q) .
\end{aligned}
$$

Proof. Since $l_{2}(x)$ is locally analytic on $x \neq 1$ and" $q^{u}$ is analytic on $|u|_{p}<$ 
$r(q) . \quad l_{2}\left(q^{u}\right)$ is locally analytic on $|u|_{p}<r(q), u \neq 0$. Thus $L_{2, q}(u)$ is locally analytic on $|u|_{p}<r(q), u \neq 0$.

Since

$$
\frac{d}{d x} l_{2}(x)=-\frac{\log (1-x)}{x} \quad \text { for } \quad x \neq 1 \text { and } x \neq 0 .
$$

Differetiating $L_{2, q}(u)$ gives the equation (2).

Using

$$
l_{2}(x)+l_{2}(1 / x)=-\frac{1}{2}(\log (x))^{2},
$$

we have the equation (3).

A simple calculation gives the equation (4).

Remark. If we define $L_{2, q}(u)$ as the function on the right hand side above then Lemma 1.1 can be proved without using Coleman [2].

We use the following lemma due to Diamond [3] to construct our $p$-adic functions.

LEMMA (1.2). Let $D$ be a subset of $\boldsymbol{C}_{p}$ such that $D+\boldsymbol{Z}_{p}$ w contained in $D$ for some $w \in C_{p}$ with $w \neq 0$. Let $b$ be a positive integer and let $f(x)$ be a locally analytic function on $D \cap\left(C_{p}-\{0\}\right)$. Define

and

$$
F(x)=\lim _{n \rightarrow \infty} \frac{1}{b p^{n}} \sum_{0 \leq j<b p n} f(x+j w) \quad \text { for } \quad x \in D \cap\left(\boldsymbol{C}_{p}-\boldsymbol{Z}_{p} w\right)
$$

$$
F^{*}(x)=\lim _{n \rightarrow \infty} \frac{1}{b p^{n}} \sum_{0 \leq j<b p^{n}}^{*} f(x+j w) \quad \text { for } \quad x \in D \cap\left(\boldsymbol{C}_{p}-Z_{p}^{*} w\right) .
$$

Then

(1) the limits exist, which are independent of $b$,

(2) $F(x)$ is locally analytic on $D \cap\left(\boldsymbol{C}_{p}-\boldsymbol{Z}_{p} w\right)$ and

(3) $F^{*}(x)$ is locally analytic on $D \cap\left(\boldsymbol{C}_{p}-\boldsymbol{Z}_{p}^{*} w\right)$.

(See Corollary of Theorem 2 of [3].)

Definition (1.3). We define analogues $G_{p, q}(x)$ and $G_{p, q}^{*}(x)$ of the Diamond's $p$-adic log-gamma functions $G_{p}(x)$ and $G_{p}^{*}(x)$ by

$$
G_{p, q}(x)=\lim _{n \rightarrow \infty} \frac{1}{p^{n}} \sum_{0 \leqq j<p} L_{2, q}(x+j) \quad \text { for } \quad x \in D(q)
$$

and

$$
G_{p, q}^{*}(x)=\lim _{n \rightarrow \infty} \frac{1}{p^{n}} \sum_{0 \leqq j<p^{n}}^{*} L_{2, q}(x+j) \quad \text { for } \quad x \in D^{*}(q)
$$

Then by Lemma (1.1) and Lemma (1.2) $G_{p, q}(x)$ and $G_{p, q}^{*}(x)$ are well-defined. And $G_{p, q}(x)$ is locally analytic on $D(q)$ and $G_{p, q}^{*}(x)$ is locally analytic on $D^{*}(q)$. 
2. Properties of $G_{p, q}(x)$ and $G_{p, q}^{*}(x)$.

In this section we investigate some properties of $G_{p, q}(x)$ and $G_{p, q}^{*}(x)$.

There is a relation between $G_{p, q}(x)$ and $G_{p, q}^{*}(x)$.

Proposition (2.1). Let $B_{1}(x)=x-(1 / 2)$ the 1-st Bernoulli polynomial.

Then

$$
G_{p, q}^{*}(x)=G_{p, q}(x)-G_{p, q p}\left(\frac{x}{p}\right)-B_{1}\left(\frac{x}{p}\right) \log \frac{1-q^{p}}{1-q}
$$

for $x \in D(q)$.

Proof. Since $|q-1|_{p}<|p|_{p}^{1 /(p-1)}$ and $r\left(q^{p}\right)=r(q) /|p|_{p}$. If $x \in D(q)$ then $x / p \in D\left(q^{p}\right)$. Thus we have

$$
\begin{aligned}
& G_{p, q}(x)-G_{p, q}\left(\frac{x}{p}\right) \\
& =\lim _{n \rightarrow \infty} \frac{1}{p^{n}} \sum_{0 \leq j<p n} L_{2, q}(x+j)-\lim _{n \rightarrow \infty} \frac{1}{p^{n-1}} \sum_{0 \leq j<p^{n-1}} L_{2, q}\left(\frac{x}{p}+j\right) \\
& =\lim _{n \rightarrow \infty} \frac{1}{p^{n}}\left\{\sum_{0 \leq j<p n} L_{2, q}(x+j)\right. \\
& \left.-\sum_{0 \leq j<p} \sum_{n-1} L_{2, q}(x+j p)+\sum_{0 \leq j<p} \sum_{n-1}(x+j p) \log \frac{1-q^{p}}{1-q}\right\} \\
& =\lim _{n \rightarrow \infty} \frac{1}{p^{n}} \sum_{0 \leq j<p^{n}}^{*} L_{2, q}(x+j)+\lim _{n \rightarrow \infty} \frac{1}{p^{n-1}} \sum_{0 \leq j \leq p^{n-1}}\left(\frac{x}{p}+j\right) \log \frac{1-q^{p}}{1-q} \\
& =G_{p, q}^{*}(x)+B_{1}\left(\frac{x}{p}\right) \log \frac{1-q^{p}}{1-q} .
\end{aligned}
$$

Because

$$
B_{1}(x)=\lim _{n \rightarrow \infty} \frac{1}{p^{n}} \sum_{0 \leq j<p n}(x+j) .
$$

Thus we complete the proof.

Remark. Koblitz [7] obtained that

$$
\phi_{p, q}^{*}(x)=\psi_{p, q}(x)-\frac{1}{p} \psi_{p, q}\left(\frac{x}{p}\right)-\frac{1}{p} \log \frac{1-q^{p}}{1-q}
$$

for $x \in D(q)$.

As for the difference equation for $\Gamma_{p, q}(x)$ Koblitz [7] obtained the following THEOREM (2.2). 


$$
\Gamma_{p, q}(x+1) / \Gamma_{p, q}(x)=\left\{\begin{array}{lll}
-\frac{1-q^{x}}{1-q} & \text { if } & x \in \boldsymbol{Z}_{p}^{*}, \\
-1 & \text { if } & x \in p \boldsymbol{Z}_{p} .
\end{array}\right.
$$

We have a difference equation for our functions $G_{p, q}(x)$ and $G_{p, q}^{*}(x)$.

THEOREM (2.3).

(1)

$$
\begin{aligned}
& G_{p, q}(x+1)-G_{p, q}(x)=\log \frac{1-q^{x}}{1-q} \quad \text { for } \quad x \in D(q) . \\
& G_{p, q}^{*}(x+p)-G_{p, q}^{*}(x)=\sum_{0<i<p} \log \frac{1-q^{x+\imath}}{1-q} \quad \text { for } \quad x \in D^{*}(q) .
\end{aligned}
$$

Remark. Koblitz [7] obtained that

$\phi_{p, q}(x+1)-\phi_{p, q}(x)=-\frac{q^{x}}{1-q^{x}} \log (q)\left(=\frac{d}{d x} \log \frac{1-q^{x}}{1-q}\right) \quad$ for $\quad x \in D(q)$.

Note that by (2) of Lemma (1.1) we have

$$
\frac{1}{p^{n}}\left\{L_{2, q}\left(x+p^{n}\right)-L_{2, q}(x)\right\}=\log \frac{1-q^{x}}{1-q}+o_{x}\left(p^{n}\right),
$$

where $o_{x}\left(p^{n}\right) \rightarrow 0(n \rightarrow \infty)$.

Proof of Theorem (2.3). By the definition we have

$$
\begin{aligned}
& G_{p, q}(x+1)-G_{p, q}(x) \\
& =\lim _{n \rightarrow \infty} \frac{1}{p^{n}}\left\{\sum_{0 \leq j<p} L_{2, q}(x+1+j)-\sum_{0 \leq j<p} L_{2, q}(x+j)\right\} \\
& =\lim _{n \rightarrow \infty} \frac{1}{p^{n}}\left\{L_{2, q}\left(x+p^{n}\right)-L_{2, q}(x)\right\} \\
& =\log \frac{1-q^{x}}{1-q} \quad \text { for } \quad x \in D(q)
\end{aligned}
$$

and

$$
\begin{aligned}
& G_{p, q}^{*}(x+p)-G_{p, q}^{*}(x) \\
& =\lim _{n \rightarrow \infty} \frac{1}{p^{n}}\left\{\sum_{0 \leq j<p n}^{*} L_{2, q}(x+p+j)-\sum_{0 \leq j<p n}^{*} L_{2, q}(x+j)\right\} \\
& =\lim _{n \rightarrow \infty} \sum_{0<i<p} \frac{1}{p^{n}}\left\{L_{2, q}\left(x+i+p^{n}\right)-L_{2, q}(x+i)\right\} \\
& =\sum_{0<i<p} \log \frac{1-q^{x+2}}{1-q} \quad \text { for } \quad x \in D^{*}(q)
\end{aligned}
$$

We have the reflection formula for our functions $G_{p, q}(x)$. Let $B_{2}(x)=x^{2}-$ 
$x+(1 / 6)$ the 2-nd Bernoulli polynomial.

THEOREM (2.4).

$$
\begin{array}{ll}
G_{p, q}(x)+G_{p, q}(1-x)=\frac{1}{2} B_{2}(x) \log (q) & \text { for } \quad x \in D(q) . \\
G_{p, q}(x)+G_{p, q^{-1}}(1-x)=-B_{1}(x) \log (q) & \text { for } \quad x \in D(q) .
\end{array}
$$

Remark. Koblitz [7] obtained that

$$
\phi_{p, q}(x)-\phi_{p, q^{-1}}(1-x)=-\log (q)=-\frac{d}{d x} B_{1}(x) \log (q) .
$$

Proof. (1) Using the definition and replacing $j$ by $p^{n}-j-1$, we have

$$
\begin{aligned}
& G_{p, q}(x)+G_{p, q}(1-x) \\
& =\lim _{n \rightarrow \infty} \frac{1}{p^{n}} \sum_{0 \leq j<p^{n}}\left\{L_{2, q}(x+j)+L_{2, q}(1-x+j)\right\} \\
& =\lim _{n \rightarrow \infty} \frac{1}{p^{n}} \sum_{0 \leqq j<p^{n}}\left\{L_{2, q}(x+j)+L_{2, q}(-x-j)\right. \\
& \left.\quad+L_{2, q}\left(-x-j+p^{n}\right)-L_{2, q}(-x-j)\right\} \quad(*) .
\end{aligned}
$$

Since (1) and (2) of Lemma (1.1), we have

$$
\frac{1}{p^{n}}\left\{L_{2, q}\left(-x-j+p^{n}\right)-L_{2, q}(-x-j)\right\}=\log \frac{1-q^{-x-\jmath}}{1-q}+o_{-x-j}\left(p^{n}\right),
$$

where $o_{-x-j}\left(p^{n}\right) \rightarrow 0(n \rightarrow \infty)$.

Using this formula and (3) of Lemma (1.1) we have

$$
\begin{aligned}
(*)= & \lim _{n \rightarrow \infty} \frac{1}{p^{n}} \sum_{0 \leq j<p^{n}}\left\{L_{2, q}(x+j)+L_{2, q}(-x-j)\right\} \\
& +\lim _{n \rightarrow \infty} p^{n} \cdot \frac{1}{p^{n}} \sum_{0 \leq j<p} \log \frac{1-q^{-x-\jmath}}{1-q}+\lim _{n \rightarrow \infty} \sum_{0 \leq j<p^{n}} O_{-x-j}\left(p^{n}\right) \\
= & \lim _{n \rightarrow \infty} \frac{1}{p^{n}} \sum_{0 \leq j<p^{n}} \frac{1}{2}(x+j)^{2} \log (q) \\
& +\lim _{n \rightarrow \infty} p^{n} \cdot \lim _{n \rightarrow \infty} \frac{1}{p^{n}} \sum_{0 \leq j<p} \log \frac{1-q^{-x-\jmath}}{1-q}+\lim _{n \rightarrow \infty} \sum_{0 \leq j<p^{n}} o_{-x-j}\left(p^{n}\right) \\
= & \frac{1}{2} B_{2}(x) \log (q) .
\end{aligned}
$$

(2) Using the definition and replacing $j$ by $p^{n}-j-1$, we have 


$$
\begin{aligned}
G_{p, q} & (x)+G_{p, q^{-1}}(1-x) \\
= & \lim _{n \rightarrow \infty} \frac{1}{p^{n}} \sum_{0 \leq j<p^{n}}\left\{L_{2, q}(x+j)+L_{2, q^{-1}}(1-x+j)\right\} \\
= & \lim _{n \rightarrow \infty} \frac{1}{p^{n}} \sum_{0 \leq j<p^{n}}\left\{L_{2, q}(x+j)+L_{2, q^{-1}}(-x-j)\right. \\
& \left.+L_{2, q^{-1}}\left(-x-j+p^{n}\right)-L_{2, q^{-1}}(-x-j)\right\} \quad(* *) .
\end{aligned}
$$

Since (1) and (2) of Lemma (1.1), we have

$$
\frac{1}{p^{n}}\left\{L_{2, q^{-1}}\left(-x-j+p^{n}\right)-L_{2, q^{-1}}(-x-j)\right\}=\log \frac{1-q^{x+j}}{1-q^{-1}}+o_{x+j}\left(p^{n}\right),
$$

where $o_{x+j}\left(p^{n}\right) \rightarrow 0(n \rightarrow \infty)$.

Then, using this formula and (4) of Lemma (1.1), we have

$$
\begin{aligned}
(* *)= & \lim _{n \rightarrow \infty} \frac{1}{p^{n}} \sum_{0 \leqq j<p^{n}}\left\{L_{2, q}(x+j)+L_{2, q^{-1}}(-x-j)\right\} \\
& +\lim _{n \rightarrow \infty} p^{n} \cdot \frac{1}{p^{n}} \sum_{0 \leqq j<p} \log \frac{1-q^{x+j}}{1-q^{-1}}+\lim _{n \rightarrow \infty} \sum_{0 \leqq j<p^{n}} o_{x+j}\left(p^{n}\right) \\
= & \lim _{n \rightarrow \infty} \frac{1}{p^{n}} \sum_{0 \leqq j<p}\{-(x+j) \log (q)\} \\
& +\lim _{n \rightarrow \infty} p^{n} \cdot \lim _{n \rightarrow \infty} \frac{1}{p^{n}} \sum_{0 \leqq j<p^{n}} \log \frac{1-q^{x+j}}{1-q^{-1}}+\lim _{n \rightarrow \infty} \sum_{0 \leqq j<p^{n}} o_{x+j}\left(p^{n}\right) \\
= & -B_{1}(x) \log (q) .
\end{aligned}
$$

The proof is completed.

We have the following corollary, which will be used in the proof of the theorem for the connection of $G_{p, q}(x)$ and $G_{p, q}^{*}(x)$ with $\Gamma_{p, q}(x)$.

COROLLARY (2.5).

$$
\sum_{0<i<p} G_{p, q}\left(\frac{i}{p}\right)=-\frac{p-1}{24} \log (q) .
$$

Proof. We have

$$
\begin{aligned}
\sum_{0<i<p} G_{p, q}\left(\frac{i}{p}\right) & =\sum_{1 \leqq \imath \leqq(p-1) / 2}\left\{G_{p, q}\left(\frac{i}{p}\right)+G_{p, q} p\left(1-\frac{i}{p}\right)\right\} \\
& =\sum_{1 \leqq \imath \leqq(p-1) / 2} \frac{1}{2} B_{2}\left(\frac{i}{p}\right) \log (q) \\
& =-\frac{p-1}{24} \log (q) .
\end{aligned}
$$

For our $G_{p, q}(x)$ and $G_{p, q}^{*}(x)$ we have the following multiplication-theorem. 
150

HAJIME NAKAZATO

THEOREM (2.6). Let $m$ be a positive integer. Then we have

$$
G_{p, q}(x)=\sum_{0 \leq i<m} G_{p, q}\left(\frac{x+i}{m}\right)+B_{1}(x) \log \frac{1-q^{m}}{1-q} \quad \text { for } \quad x \in D(q),
$$

and when $m \equiv 0(\bmod p)$ we have

(2)

$$
G_{p, q}^{*}(x)=\sum_{0 \leq i<m}^{*} G_{p, q^{m}}\left(\frac{x+i}{m}\right)+\left(1-\frac{1}{p}\right) x \cdot \log \frac{1-q^{m}}{1-q} \quad \text { for } \quad x \in D^{*}(q) .
$$

Remark. Koblitz [7] obtained that

$$
\phi_{p, q}(x)=\frac{1}{m} \sum_{0 \leq i<m} \psi_{p, q}\left(\frac{x+i}{m}\right)+\log \frac{1-q^{m}}{1-q} \quad \text { for } \quad x \in D(q) .
$$

Proof. (1) By the definition we have for $x \in D(q)$

$$
\begin{aligned}
& \sum_{0 \leq i<m} G_{p, q m}\left(\frac{x+i}{m}\right) \\
& =\lim _{n \rightarrow \infty} \frac{1}{p^{n}} \sum_{0 \leq i<m} \sum_{0 \leq j<p^{n}}\left\{-\frac{1}{\log \left(q^{m}\right)} l_{2}\left(q^{x+\imath+\jmath m}\right)-\left(\frac{x+i}{m}+j\right) \log \left(1-q^{m}\right)\right\} \\
& =\lim _{n \rightarrow \infty} \frac{1}{m p^{n}} \sum_{0 \leqq \jmath<m}\left\{L_{2, q}(x+j)-(x+j) \log \frac{1-q^{m}}{1-q}\right\} \\
& =G_{p, q}(x)-B_{1}(x) \log \frac{1-q^{m}}{1-q} .
\end{aligned}
$$

(2) By the definition we have for $x \in D^{*}(q)$

$$
\begin{aligned}
& \sum_{0 \leq i<m} G_{p, q m}\left(\frac{x+i}{m}\right) \\
& =\lim _{n \rightarrow \infty} \frac{1}{p^{n}} \sum_{0 \leq i<m}^{*} \sum_{0 \leq j<p^{n}}\left\{-\frac{1}{\log \left(q^{m}\right)} l_{2}\left(q^{x+\imath+j m}\right)-\left(\frac{x+i}{m}+j\right) \log \left(1-q^{m}\right)\right\} \\
& =\lim _{n \rightarrow \infty} \frac{1}{m p^{n}} \sum_{0 \leq j<m p^{n}}^{*}\left\{L_{2, q}(x+j)-(x+j) \log \frac{1-q^{m}}{1-q}\right\} \\
& =G_{p, q}^{*}(x)-\left(B_{1}(x)-B_{1}\left(\frac{x}{p}\right)\right) \log \frac{1-q^{m}}{1-q} \\
& =G_{p, q}^{*}(x)-\left(1-\frac{1}{p}\right) x \cdot \log \frac{1-q^{m}}{1-q} .
\end{aligned}
$$

Letting $m=p$ we have the following

COROLLARY (2.7).

$$
G_{p, q}^{*}(x)=\sum_{0<i<p} G_{p, q} p\left(\frac{x+i}{p}\right)+\left(1-\frac{1}{p}\right) x \cdot \log \frac{1-q^{p}}{1-q} \quad \text { for } \quad x \in D^{*}(q) .
$$


Letting $x=0$ and using Corollary (2.5) we have the following

COROLLARY (2.8).

$$
G_{p, q}^{*}(0)=\sum_{0<i<p} G_{p, q}\left(\frac{i}{p}\right)=-\frac{p-1}{24} \log (q) .
$$

\section{Connections with $\Gamma_{p, q}(x)$.}

In this section we study the connections of $G_{p, q}(x)$ and $G_{p, q}^{*}(x)$ with $\Gamma_{p, q}(x)$. By the definition of $\Gamma_{p, q}(x)$ [7] we have

$$
\Gamma_{p, q}(1)=-1 \text {. }
$$

Then the difference equation of Theorem (2.2) follows

Thus we have

$$
\Gamma_{p, q}(0)=1 \text {. }
$$

$$
\log \Gamma_{p, q}(0)=0 .
$$

By Theorem (2.2) we have

$$
\log \Gamma_{p, q}(x+1)-\log \Gamma_{p, q}(x)=\left\{\begin{array}{lll}
\log \frac{1-q^{x}}{1-q} & \text { if } \quad x \in \boldsymbol{Z}_{p}^{*}, \\
0 & \text { if } \quad x \in p \boldsymbol{Z}_{p} .
\end{array}\right.
$$

Using Corollary (2.5) we have

$$
\sum_{0<i<p}\left\{G_{p, q} p\left(\frac{i}{p}\right)-\frac{\log (q)}{24}\right\}=0
$$

Then the connections of $G_{p, q}(x)$ and $G_{p, q}^{*}(x)$ with $\Gamma_{p, q}(x)$ are the following THEOREM (3.1).

$$
\begin{aligned}
& \log \Gamma_{p, q}(x)=G_{p, q}^{*}(x)+\frac{p-1}{24} \log (q) \quad \text { for } \quad x \in p \boldsymbol{Z}_{p} . \\
& \log \Gamma_{p, q}(x) \\
& =\sum_{\substack{0 \leq i \leq p \\
x+i \in \mathbb{Z}_{p}^{*}}}\left\{G_{p, q}\left(\frac{x+i}{p}\right)+\frac{\log (q)}{24}\right\}+(x-\tilde{x}) \log \frac{1-q^{p}}{1-q} \quad \text { for } \quad x \in \boldsymbol{Z}_{p} .
\end{aligned}
$$

Remark. Koblitz [7] obtained that
(1) $\frac{d}{d x} \log \Gamma_{p, q}(x)=\phi_{p, q}^{*}(x) \quad$ for $\quad x \in p \boldsymbol{Z}_{p}$,
(2) $\frac{d}{d x} \log \Gamma_{p, q}(x)=\frac{1}{p} \sum_{\substack{0 \leq i \leq p \\ x+i \in \mathbf{Z}_{p}^{*}}} \psi_{p, q}\left(\frac{x+i}{p}\right)+\left(1-\frac{1}{p}\right) \log \frac{1-q^{p}}{1-q} \quad$ for $\quad x \in \boldsymbol{Z}_{p}$. 
Proof of Theorem (3.1). Since both sides of (2) are continuous in $x \in Z_{p}$, it suffices to prove (2) for $x=$ any non-negative integer $n$.

Let $A_{n}$ denote the left side of (2) for $x=n$, and let $B_{n}$ denote the right side of (2) for $x=n$. We prove $A_{n}=B_{n}$ by induction $n$.

Note that by (i) and (iii) we have $A_{0}=B_{0}$. Suppose that $A_{n}=B_{n}$.

By (ii) we have

$$
A_{n+1}-A_{n}=\left\{\begin{array}{lll}
\log \frac{1-q^{n}}{1-q} & \text { if } & n \neq 0(\bmod p), \\
0 & \text { if } & n \equiv 0(\bmod p)
\end{array}\right.
$$

We claim that

$$
B_{n+1}-B_{n}=\left\{\begin{array}{lll}
\log \frac{1-q^{n}}{1-q} & \text { if } & n \neq 0(\bmod p), \\
0 & \text { if } & n \equiv 0(\bmod p) .
\end{array}\right.
$$

In fact we have

$$
\begin{aligned}
& B_{n+1}-B_{n} \\
& =\sum_{\substack{0 \leq i<p \\
n+1+i \neq 0(\bmod p)}}\left\{G_{p, q p}\left(\frac{n+1+i}{p}\right)+\frac{\log (q)}{24}\right\} \\
& \quad+\sum_{\substack{n \leq i<p \\
n+\imath \neq 0(\bmod p)}}\left\{G_{p, q p}\left(\frac{n+i}{p}\right)+\frac{\log (q)}{24}\right\} \\
& \quad+\left\{(n+1)-(n+1)^{\sim}-n+\tilde{n}\right\} \log \frac{1-q^{p}}{1-q} \\
& =\sum_{n+1+i<p(\bmod p)} G_{p, q p}\left(\frac{n+1+i}{p}\right)+\sum_{\substack{0 \leq i<p \\
n+i \neq 0(\bmod p)}} G_{p, q p}\left(\frac{n+i}{p}\right) \\
& \quad+\{[(n-1) / p]+1-[n / p]\} \log \frac{1-q^{p}}{1-q} \cdots \cdots(*) .
\end{aligned}
$$

(a) Case of $n \neq \equiv(\bmod p)$. We have

$$
\begin{aligned}
(*) & =G_{p, q p}\left(\frac{n+p}{p}\right)-G_{p, q}\left(\frac{n}{p}\right)+\log \frac{1-q^{p}}{1-q} \\
& =\log \frac{1-q^{n}}{1-q^{p}}+\log \frac{1-q^{p}}{1-q}=\log \frac{1-q^{n}}{1-q} .
\end{aligned}
$$

(b) Case of $n \equiv 0(\bmod p)$. We have

By (a) and (b) we have

$$
(*)=0 \text {. }
$$

and so we have

$$
A_{n+1}-A_{n}=B_{n+1}-B_{n},
$$

$$
A_{n+1}=B_{n+1} \text {. }
$$


This completes the induction.

To prove (1) we use Corollary (2.7) and the following formula

$$
x-\tilde{x}=\left(1-\frac{1}{p}\right) \cdot x \quad \text { for } \quad x \in p Z_{p} .
$$

By (2) for $x \in p \boldsymbol{Z}_{p}$, we have

$$
\begin{aligned}
& \log \Gamma_{p, q}(x) \\
& =\sum_{0 \leq 2<p}\left\{G_{p, q p}\left(\frac{x+i}{p}\right)+\frac{\log (q)}{24}\right\}+(x-\tilde{x}) \log \frac{1-q^{p}}{1-q} \\
& =\sum_{0 \leq i<p} G_{p, q p}\left(\frac{x+i}{p}\right)+\left(1-\frac{1}{p}\right) x \cdot \log \frac{1-q^{p}}{1-q}+\frac{p-1}{24} \log (q) \\
& =G_{p, q}^{*}(x)+\frac{p-1}{24} \log (q) .
\end{aligned}
$$

The proof is completed.

\section{REFERENCES}

[1] P. CAssou-Nogues: Applications arithmetiques de l'etude des valeurs aux entiers negatifs des series de Dirichlet associees a un polynome, Ann. Inst. Fourier, Grenoble 31, 4 (1981), 1-35.

[2] R.F. Coleman, Dilogarithms, Regulators and $p$-adic $L$-functions, invent. math., 69 (1982), 171-208.

[3] J. Diamond, The p-adic log gamma function and p-adic Euler constants, Trans. Amer. Math. Soc., 233 (1977), 321-337.

[4] B. Ferrero and R. Greenberg, On the behavior of $p$-adic $L$-functions at $s=0$, invent. math., 50 (1978), 91-102.

[5] K. Iwasawa, Lectures on $p$-adic $L$-functions, Princeton Univ. Press, Princeton, N. J., 1972.

[6] N. Koblitz, p-adic analysis: A short course on recent work, London Math. Soc. Lecture Note Series 46, Cambridge Univ. Press, Cambridge, 1980.

[7] N. Koblitz, $q$-extensions of the p-adic gamma function, Trans. Amer. Math. Soc., 260 (1980), 449-457.

[8] N. Koblitz, $q$-extensions of the p-adic gamma function II, ibid., 273 (1982), $111-129$.

[9] Y. Morita, A $p$-adic analogue of the $\Gamma$-function, J. Fac. Sc. Univ. Tokyo Sect. IA Math., 22 (1975), 255-266.

Department of Mathematics

Tokyo National College of Technology

1220-2 Kunugida-Machi, HachioJi

TOKYO, 193 JAPAN 
